\title{
Modelagem robusta para a satisfação de clientes de serviços
}

\author{
Maria Auxiliadora Cannarozzo Tinoco ${ }^{\mathrm{a} *}$, José Luis Duarte Ribeiro ${ }^{\mathrm{b}}$ \\ a*maria@producao.ufrgs.br, UFRGS, Brasil \\ bribeiro@producao.ufrgs.br, UFRGS, Brasil
}

\begin{abstract}
Resumo
0 presente artigo apresenta a proposição de uma abordagem robusta para a modelagem da satisfação de clientes de serviços. A abordagem robusta representa uma extensão da abordagem proposta por Tinoco e Ribeiro (2007), através da inclusão de estatísticas robustas que podem representar adequadamente as relações causais que envolvem a satisfação de clientes de serviços, mesmo na presença de respondentes inconsistentes. Os procedimentos robustos permitem identificar esses respondentes inconsistentes e atribuir uma ponderação em função da concordância de cada questionário em relação ao conjunto das observações. A modelagem proposta é apresentada e testada através da construção de um modelo geral de satisfação de clientes de serviços. Para isso, foram avaliados 12 segmentos de serviços escolhidos para o estudo por contemplarem diferentes categorias de serviços, no que se relaciona a grau tangibilidade e grau de contato e de envolvimento do cliente. A abordagem robusta permitiu confirmar a maioria das relações significativas entre determinantes da satisfação obtidas a partir da abordagem tradicional proposta por Tinoco e Ribeiro (2007) e, além disso, incorporar ao modelo outra relação significativa que não seria considerada a partir da técnica tradicional.
\end{abstract}

Palavras-chave

Modelagem robusta. Satisfação de clientes. Serviços.

\section{Introdução}

A satisfação do cliente é um conceito que tem recebido muita atenção e interesse por parte de pesquisadores e empresários nos últimos anos. lsso, motivado pela sua importância como elemento chave nas estratégias de negócios das empresas que procuram sobreviver e ganhar vantagem competitiva na economia atual e pelo seu papel no desempenho das organizações públicas e privadas (CARO; GARCÍA, 2007).

Devido à sua importância, muitas pesquisas da literatura vêm estudando os principais determinantes da satisfação de clientes e o seu relacionamento causal. Existem diversas abordagens utilizadas para modelar essas relações entre determinantes. Uma das técnicas mais utilizadas para a modelagem de relações causais entre determinantes da satisfação de clientes é a análise de equações estruturais. Contudo, esse método apresenta algumas limitações de caráter prático, entre elas a necessidade de tamanhos de amostras grandes e cada vez maiores, à medida que os modelos se tornam complexos (GOSLING; GONÇALVES, 2003; MEYERS; GAMST; GUARINO, 2006), e a necessidade de uma base de dados quantitativa. Para superar essas limitações, Tinoco e Ribeiro (2007) propõem uma abordagem para a construção de modelos causais de satisfação dos clientes de serviços, seguindo procedimentos mais simples, possibilitando a modelagem de múltiplos determinantes e sem a necessidade de grandes tamanhos de amostra.

Uma vez que a abordagem proposta por Tinoco e Ribeiro (2007) permite identificar relações entre múltiplos determinantes da satisfação de clientes a partir do uso de tamanhos de amostras relativamente pequenos, a presença de dados atípicos, que se afastam da média das observações, pode distorcer a análise. Nesse sentido, é pertinente a incorporação de procedimentos robustos que minimizem o efeito de eventuais dados atípicos na abordagem proposta por 
Tinoco e Ribeiro (2007). Os procedimentos robustos, geralmente utilizados na análise de regressão, tentam extrair a melhor informação possível dos dados, através da identificação do padrão que melhor se ajusta à maioria das observações.

Nesse contexto, o presente artigo tem por objetivo propor uma abordagem robusta para a modelagem da satisfação de clientes de serviços a partir da sistemática proposta por Tinoco e Ribeiro (2007). A abordagem proposta faz uso de estatísticas robustas para gerar modelos que representem adequadamente a realidade estudada, mesmo frente a algum percentual de dados atípicos. A modelagem robusta proposta neste trabalho é apresentada e testada através da construção de um modelo geral de satisfação de clientes de serviços.

A seguir, a seção 2 apresenta o referencial teórico, abordando assuntos relacionados com modelos de satisfação de clientes e estimação robusta. Na terceira seção é apresentada a modelagem robusta proposta. A seção 4 apresenta a aplicação da abordagem proposta na construção de um modelo geral de serviços. Finalmente, a seção 5 aborda as conclusões do artigo.

\section{Referencial teórico}

\subsection{Modelos de satisfação de clientes}

Diversos estudos na literatura estão focados na proposição de modelos que representam a satisfação dos clientes com produtos e serviços. Isso devido à importância cada vez mais crescente da satisfação dos clientes para as organizações e nações. Pesquisas apontam que a satisfação é um dos principais determinantes das intenções futuras dos clientes (CRONIN JUNIOR; BRADY; HULT, 2000; WANG; LO, 2002; CHOI et al., 2004; BIGNÉ; MATTILA; ANDREU, 2008; MARTIN et al., 2008; VLACHOS; VRECHOPOULOS, 2008). A satisfação do cliente também é considerada um indicativo da sua lealdade a uma determinada empresa e marca de produto (ANDREASSEN; LINDESTAD, 1998; NGUYEN; LEBLANC, 1998; JOHNSON; GUSTAFSSON, 2000; BEl; CHIAO, 2001; MORGAN; REGO, 2006). Além disso, estudos têm mostrado que a maior satisfação do cliente leva a melhores resultados financeiros (e.g., ANDERSON; FORNELL, 2000; ANDERSON et al., 1994; BRUHN; GRUND, 2000; JOHNSON; GUSTAFSSON, 2000). Essas são algumas das razões que justificam o interesse de acadêmicos e empresas em estudar, definir e medir a satisfação do cliente.

Grande parte dos modelos de satisfação de clientes da literatura está fundamentada no paradigma da desconfirmação de expectativas como o principal determinante da satisfação do cliente (e.g., OLIVER, 1980, 1993; OLIVER; RUST; VARKI, 1997; CHURCHILL JUNIOR; SURPRENANT, 1982; TSE; WILTON, 1988; Yl, 1993; JOHNSON; NADER; FORNELL, 1996; SPRENG; MACKENZIE; OLSHAVSKY, 1996; SPRENG; MACKOY, 1996; JUN; HYUN; GENTRY, 2001; SZYMANSKI; HENARD, 2001; TSIROS; MITTAL; ROSS JUNIOR, 2004; CARO; GARCÍA, 2007). Isso ocorre porque o modelo de desconfirmação de expectativas é considerado a teoria dominante na avaliação global da satisfação de clientes. Assim, diversas pesquisas têm documentado a crescente aceitação desse paradigma para descrever a avaliação pós-compra dos consumidores (SZYMANSKI; HENARD, 2001).

Oliver (1980) foi um dos primeiros autores a incluir a desconfirmação de expectativas em um modelo de satisfação do cliente. De acordo com o autor, sentimentos de satisfação surgem quando clientes comparam suas percepções do desempenho do produto às suas expectativas.

Outros pesquisadores (e.g., CHURCHILL JUNIOR; SURPRENANT, 1982; TSE; WILTON, 1988; Yl, 1993; JOHNSON; NADER; FORNELL, 1996; TSIROS; MITTAL; ROSS JUNIOR, 2004) avaliaram o modelo de desconfirmação de expectativas proposto por Oliver (1980), obtendo resultados diferentes em relação à influência direta das expectativas, do desempenho percebido e da desconfirmação de expectativas na satisfação. As diferenças encontradas pelos autores podem ser explicadas pelos diferentes contextos de produtos e serviços avaliados.

As bases afetivas da satisfação não estão bem documentadas na literatura (OLIVER, 2010). As emoções do consumidor não foram plenamente reconhecidas nos estudos de satisfação do cliente (GRÖNROOS, 2007; CARO; GARCÍA, 2007). Contudo, alguns autores concordam que reações emocionais associadas com uma experiência de consumo são importantes na construção da satisfação (OLIVER, 1993, 2010; OLIVER; RUST; VARKI, 1997; JUN; HYUN; GENTRY, 2001). A influência das emoções na satisfação de clientes é particularmente significativa em contextos de serviços hedônicos (e.g., JIANG; WANG, 2006; CARO; GARCÍA, 2007; MARTIN et al., 2008; BIGNÉ; MATTILA; ANDREU, 2008), nos quais a experiência de consumo é influenciada principalmente por aspectos emotivos (JIANG; WANG, 2006).

Outras pesquisas (e.g., SPRENG; MACKENZIE; OLSHAVSKY, 1996; SPRENG; MACKOY, 1996; OLSHAVSKY; KUMAR, 2001) consideram o grau no qual um produto ou serviço atende os desejos do cliente como um importante determinante da satisfação. Nesse caso, a comparação entre as percepções de desempenho e desejos pessoais dos clientes podem influenciar na sua satisfação. 
A qualidade percebida e o valor percebido têm surgido também como importantes determinantes da satisfação de clientes na literatura (e.g., CRONIN JUNIOR; BRADY; HULT, 2000; BEI; CHIAO, 2001; WANG; LO, 2002; CHOl et al., 2004; COLLIER; BIENSTOCK, 2006; WHITTAKER; LEDDEN; KALAFATIS, 2007; VLACHOS; VRECHOPOULOS, 2008). Contudo, algumas pesquisas demonstram que a satisfação do cliente está vinculada à percepção de valor em muitas mas não em todas as transações (DAY, 2002).

A imagem corporativa constitui outro dos determinantes da satisfação encontrados na literatura. Pesquisas sugerem que a imagem corporativa exerce uma grande influência na satisfação dos clientes, principalmente em serviços complexos, onde os atributos de qualidade são difíceis de avaliar (ANDREASSEN; LINDESTAD, 1998). Em uma relação inversa, a satisfação de clientes também pode influenciar a imagem corporativa de uma empresa de serviços (NGUYEN; LEBLANC, 1998).

A percepção do cliente em relação ao preço também é considerada determinante da satisfação do cliente (BEI; CHIAO, 2001; HERRMANN et al., 2007). Quando o cliente percebe que o preço de um produto ou serviço é razoável, ele vai sentir maior satisfação manifestando a sua intenção de repetir a compra (BEl; CHIAO, 2001). Portanto, a percepção de justiça no preço pago do ponto de vista do cliente pode influenciar a sua satisfação (LIANG; ZHANG, 2009). A relação inversa, o impacto da satisfação do cliente sobre a percepção de preço, também pode ser verificada na literatura. Isto acontece quando uma maior satisfação do cliente com um produto ou serviço diminui o impacto negativo de um aumento de preços (HOMBURG; HOYER; KOSCHATE, 2005).

Outros fatores cognitivos tais como equidade e atribuições têm sido propostos também como determinantes da satisfação, mas não geraram o mesmo nível de interesse nas pesquisas de satisfação do cliente. Considera-se que, geralmente, os clientes ficam mais satisfeitos quando percebem tratamento justo ou equidade e quando atribuem resultados favoráveis às ações deles mesmos e resultados não favoráveis aos outros (OLIVER, 1993, 2010).

Em conclusão, estudos da literatura apontam como principais determinantes da satisfação de clientes a desconfirmação de expectativas e a qualidade percebida pelos clientes (conforme Quadro 1). Outras variáveis importantes consideradas nos estudos são as expectativas, as emoções dos clientes, o valor e o desempenho percebido. A imagem corporativa, os desejos dos clientes e o preço percebido também são apontados, com menor frequência, como determinantes diretos da satisfação de clientes. Embora nenhum modelo da literatura apresente todos os determinantes em si, existe a necessidade de avaliar todas as possíveis variáveis que possam influenciar no processo de formação da satisfação de clientes, uma vez que essas variáveis constituem fatores cognitivos e afetivos dos clientes, importantes na formação de atitudes e intenções de consumo de produtos e serviços.

0 Quadro 1 apresenta um resumo dos principais modelos de satisfação de clientes encontrados na literatura, destacando os determinantes diretos da satisfação e, além disso, outras relações entre determinantes propostas nos modelos. As relações são mostradas através de flechas que indicam o sentido da influência.

\subsection{Estimação robusta}

Eventualmente, um conjunto de dados pode possuir algumas observações discordantes do resto da amostra estudada. Essas observações que se distanciam do conjunto de dados são chamadas de outliers ou dados atípicos e podem exercer grande influência na análise dos resultados. A presença de outliers pode conduzir a resultados distorcidos. Assim, a validade de conclusões derivadas de análises estatísticas depende, muitas vezes, da identificação e remoção e tratamento adequado dos potenciais outliers (ALMA, 2011; HUBER; RONCHETTI, 2009; ABRAMOVITZ; MENDES, 2000; ANALYTICAL..., 1989; KLEINBAUM; KUPPER, 1978).

Dada uma amostra, inúmeras causas estão associadas ao surgimento de outliers. Na modelagem da satisfação de clientes, o surgimento de dados discrepantes pode resultar da falta de interesse do respondente, falta de compreensão do instrumento de coleta de dados, erros de digitação de dados, entre outros. Portanto, a aplicação de procedimentos robustos no tratamento dos dados pode minimizar os efeitos negativos da presença de dados atípicos.

Existem duas formas básicas de tratar dados atípicos ou outliers. A primeira delas consiste em identificar esses outliers e eliminá-los da amostra. A segunda forma reside na aplicação de procedimentos robustos que irão ponderar adequadamente os dados suspeitos, através da aplicação de testes de discrepância (KLEINBAUM et al., 2008; BUSTOS, 1988; MONTGOMERY; PECK; VINING, 2006). Neste trabalho, a modelagem robusta considera a aplicação de estatísticas robustas para minimizar os efeitos dos dados atípicos sem eliminá-los da amostra.

\section{Modelagem robusta proposta}

A abordagem proposta neste trabalho para a modelagem da satisfação de clientes representa uma 
Quadro 1. Determinantes da satisfação do cliente encontrados na literatura.

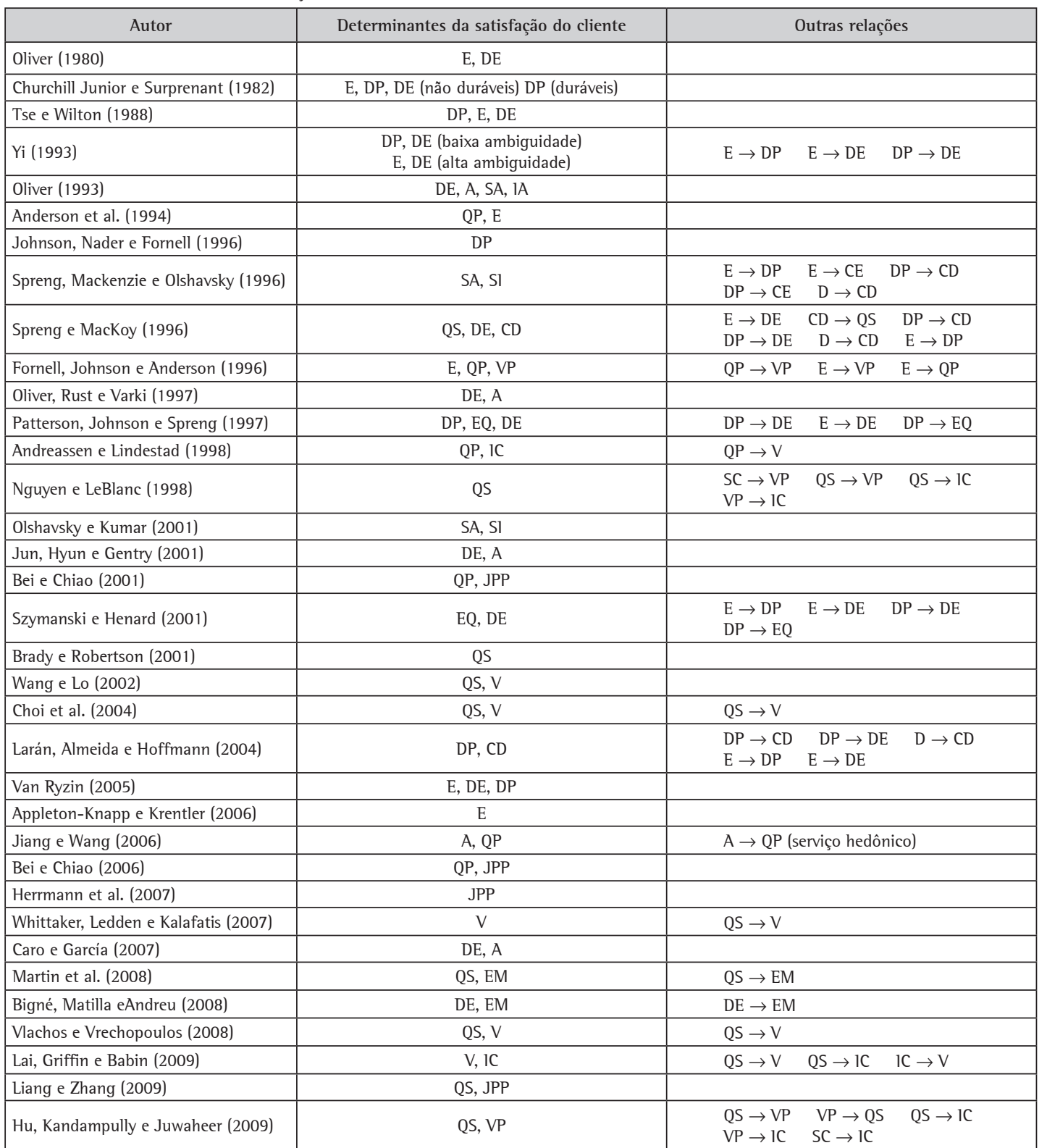

A: afetos; CD: congruência de desejos; CE: congruência de expectativas; D: desejos; DE: desconfirmação de expectativas; DP: desempenho percebido; E: expectativas; EM: emoções; EQ: equidade; IA: insatisfação com atributos; IC: imagem corporativa; JPP: justiça de preço percebido; QP: qualidade percebida; QS: qualidade do serviço; SA: satisfação com atributos; SC: satisfação do consumidor; SI: satisfação com a informação; V: valor; VP: valor percebido.

extensão do trabalho de Tinoco e Ribeiro (2007) através da inclusão de procedimentos robustos. A modelagem robusta apresenta as mesmas vantagens em relação aos métodos tradicionais de modelagem, como, por exemplo, as equações estruturais, uma vez que: (i) possibilita a identificação de relações entre múltiplos determinantes sem necessidade de um tamanho de amostra muito grande; (ii) utiliza procedimentos estatísticos muito mais simples, baseados fundamentalmente na análise de resíduos padronizados, para a identificação de relações significativas; e (iii) permite minimizar o erro de especificação no processo de modelagem, visto que podem ser consideradas todas as variáveis independentes de efeito relevante sem tornar o modelo muito complexo.

Além disso, a nova abordagem considera estatísticas robustas que podem representar adequadamente as relações causais que envolvem a satisfação de clientes de serviços, mesmo na presença 
de dados atípicos. No âmbito deste trabalho, dados atípicos correspondem a respondentes cujas respostas estão afastadas daquelas fornecidas pela maioria. A modelagem robusta proposta é constituída pelas etapas apresentadas na Figura 1.

As primeiras quatro etapas da modelagem robusta proposta neste estudo estão fundamentadas na abordagem proposta por Tinoco e Ribeiro (2007) para a construção de modelos de satisfação de clientes de serviços. A extensão dessa abordagem acontece na última etapa da construção do modelo, onde são acrescentados os passos 3.5.1 a 3.5.3, com a finalidade de gerar um modelo robusto resistente à influência de respondentes inconsistentes. A seguir são detalhadas as etapas da modelagem robusta proposta neste trabalho e sua aplicação para a construção de um modelo geral de satisfação de clientes de serviços.

\subsection{Definição dos determinantes para a construção do modelo}

Nesta etapa são definidos os determinantes a serem incluídos no modelo, a respeito dos quais são elaboradas as questões do instrumento de pesquisa. Tinoco e Ribeiro (2007) realizaram um estudo dos modelos de satisfação dos consumidores encontrados na literatura e identificaram os principais determinantes que afetam diretamente a satisfação do cliente e que são considerados na maioria das pesquisas. No presente estudo, foram selecionados os mesmos determinantes propostos por Tinoco e Ribeiro (2007),

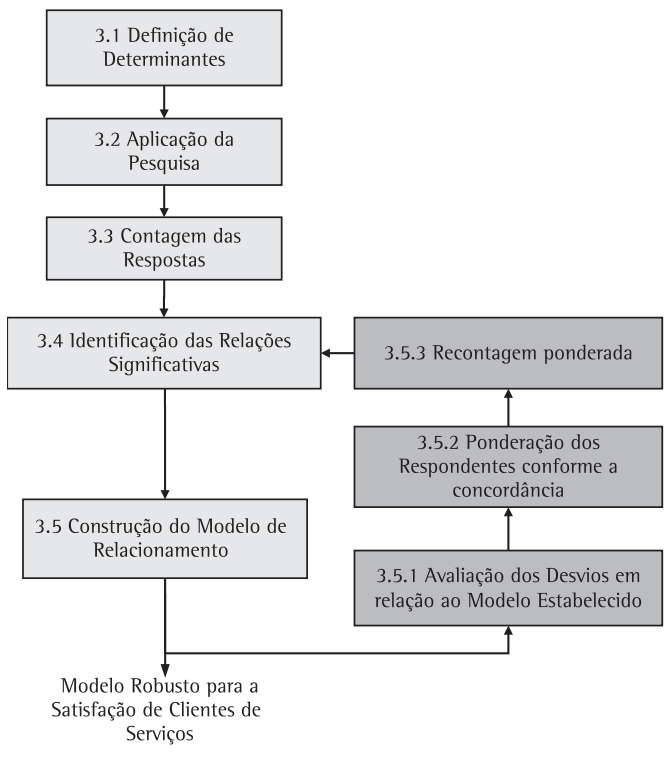

Figura 1. Etapas da modelagem robusta para a satisfação dos clientes de serviços. uma vez que contemplam as principais variáveis encontradas na literatura, com algumas exceções e adaptações semânticas. Isso foi confirmado da análise dos modelos da literatura apresentada no Quadro 1 e do Quadro 2.

No Quadro 2, as variáveis qualidade do serviço e desempenho percebido, da literatura, foram agrupadas no determinante qualidade percebida, representando a avaliação geral da excelência do serviço do ponto de vista do cliente. Da mesma forma, valor do serviço foi utilizado em lugar de valor percebido; emoções vivenciadas em lugar de afetos e emoções; preço percebido em lugar de justiça do preço percebido e equidade. Para fins da aplicação da pesquisa, no questionário foi utilizado confirmação de expectativas em lugar de desconfirmação de expectativas. Essas adaptações semânticas foram realizadas para facilitar o entendimento dos respondentes na pesquisa. A influência dos desejos dos clientes na satisfação com serviços é verificada incluindo-se na modelagem a variável desejos pessoais em lugar da congruência de desejos; e a (in)satisfação com os atributos e com a informação é considerada dentro do construto satisfação dos clientes.

Importante esclarecer que os estudos encontrados na literatura não contemplam todos os determinantes em um modelo único. Isso acontece porque, geralmente, a metodologia utilizada envolve o emprego de equações estruturais, o que implica a exigência de medidas quantitativas de todos os determinantes mencionados, além de uma extensa base de dados que permita a avaliação da existência de relação

Quadro 2. Determinantes da satisfação dos clientes utilizados no estudo.

\begin{tabular}{|c|c|}
\hline $\begin{array}{c}\text { Determinantes } \\
\text { da literatura }\end{array}$ & $\begin{array}{c}\text { Determinantes no } \\
\text { presente estudo }\end{array}$ \\
\hline $\begin{array}{l}\text { Desconfirmação de } \\
\text { expectativas (DE) }\end{array}$ & $\begin{array}{l}\text { Desconfirmação } \\
\text { de expectativas }\end{array}$ \\
\hline Qualidade do serviço (QS) & \multirow{3}{*}{ Qualidade percebida } \\
\hline Qualidade percebida (QP) & \\
\hline Desempenho percebido (DP) & \\
\hline Expectativas (E) & Expectativas \\
\hline Valor (V) & \multirow{2}{*}{ Valor do serviço } \\
\hline Valor percebido (VP) & \\
\hline Afetos (A) & \multirow{2}{*}{ Emoções vivenciadas } \\
\hline Emoções (EM) & \\
\hline Justiça do preço percebido (JPP) & \multirow{2}{*}{ Preço percebido } \\
\hline Equidade (EQ) & \\
\hline Imagem corporativa (1C) & Imagem corporativa \\
\hline Congruência de desejos (CD) & Desejos pessoais \\
\hline Satisfação com atributos (SA) & \multirow{3}{*}{ Satisfação do cliente } \\
\hline Satisfação com a informação (SI) & \\
\hline Insatisfação com atributos (IA) & \\
\hline
\end{tabular}


entre determinantes. Portanto, a grande maioria dos modelos pesquisados está focada no estudo de alguns poucos determinantes, considerados os mais importantes (ou referente aos quais foi possível reunir dados quantitativos), que afetam a satisfação dos consumidores.

Importante mencionar que o método proposto pode ser empregado com outro conjunto de determinantes, que podem ser mais representativos de um determinado setor de serviço ou constituir o objeto de interesse em uma pesquisa mais específica.

\subsection{Aplicação da pesquisa}

Nesta etapa é realizado o levantamento dos dados através da aplicação de uma pesquisa descritiva, tendo como finalidade identificar as relações entre os determinantes selecionados na etapa anterior. 0 instrumento de coleta de dados é constituído pelo questionário de nove perguntas proposto por Tinoco e Ribeiro (2007). Cada questão pergunta a dependência de cada uma das variáveis estudadas (desejos pessoais, expectativas, emoções vivenciadas no momento da prestação, qualidade percebida, preço percebido, valor do serviço, desconfirmação de expectativas, imagem corporativa e satisfação dos clientes) em relação às outras. As questões podem ser respondidas utilizando múltipla escolha ou selecionando nenhuma das alternativas.

Em função da variabilidade presente nas respostas, observou-se que cerca de 50 respondentes são suficientes para a estruturação do modelo de satisfação para um serviço em específico, com a precisão desejada (TINOCO; RIBEIRO, 2007). Utilizando esse tamanho de amostra são atendidas as condições necessárias para a aplicação do teste de qui-quadrado (frequências maiores de 5 em pelo menos $80 \%$ das células) (AGRESTI; FINLAY, 2009). Além disso, é possível detectar frequências significativas (contagens) no mínimo 40\% maiores que a média.

\subsection{Contagem das respostas}

Partindo da coleta dos dados dos questionários, identifica-se o total de respondentes que assinalou a existência de uma determinada relação entre determinantes. Os valores observados (contagens) são agrupados em uma matriz, onde o cabeçalho das linhas é constituído pelos determinantes, entendidos como variáveis dependentes, e o cabeçalho das colunas é constituído pelos mesmos determinantes, entendidos como variáveis independentes.

\subsection{Identificação das relações significativas}

Para determinar quais das relações entre os determinantes são significativas, realiza-se a análise dos resíduos padronizados. Os resíduos padronizados são calculados a partir da seguinte equação (AGRESTI; FINLAY, 2009):

$$
r_{i j}=\frac{f o_{i j}-f e}{\sqrt{f e\left(1-\% d a \text { linha }_{i}\right)\left(1-\% \text { coluna }_{j}\right)}}
$$

onde

- $r_{\mathrm{ij}}$ : resíduo padronizado;

- $\mathrm{fo}_{\mathrm{ij}}$ : valor observado (no caso, a contagem obtida para cada determinante);

- fe: valor esperado (no caso, a média dos valores observados).

0 percentual da linha é calculado somando-se os valores das contagens para cada variável dependente (cabeçalho das linhas) e dividindo-se esse valor pela soma total das contagens. 0 percentual da coluna é calculado da mesma forma, porém utilizando-se as contagens das variáveis independentes (cabeçalho das colunas).

A análise de resíduos padronizados fornece informação em relação aos valores que se afastam do esperado, podendo ser utilizada para determinar a intensidade das relações correspondentes. Esses resíduos padronizados se comportam como a variável reduzida z. Dessa forma, os resíduos padronizados indicam quantos desvios padrões uma contagem qualquer está afastada da contagem média observada ao longo de todas as células. Um valor elevado de resíduo padronizado fornece evidência contra a hipótese nula (AGRESTI; FINLAY, 2009), que neste estudo estabelece que todas as relações têm aproximadamente a mesma intensidade.

$\mathrm{Na}$ modelagem da satisfação do cliente, em princípio, todos os determinantes exercem alguma influência sobre os demais. 0 objetivo é identificar as principais relações (relações fortes) que existem entre os determinantes envolvidos. Os resíduos padronizados resultam igual a 0 quando a contagem é igual ao valor médio das contagens observadas em todas as células. Por outro lado, quando o valor do resíduo padronizado alcança e supera $+1,96$ (nível de significância associado a 0,05), observa-se uma evidência moderada de que a relação em questão é significativamente mais forte do que a média. Por fim, quando o valor do resíduo padronizado alcança e supera +2,67 (nível de significância associado a 0,01), observa-se uma evidência forte de que a relação em questão é significativamente mais intensa 
do que o relacionamento médio que existe entre os determinantes.

Portanto, utilizando-se os desvios padronizados é possível identificar-se as relações significativas entre os determinantes do modelo. Os valores significativos representam evidência estatística da existência de relação entre os determinantes em estudo. Para fins desta pesquisa, o modelo geral de serviços considera como critério de decisão valores de desvio maiores que $+2,67$. Esse valor de desvio está associado a um nível de significância alfa igual a 0,01. Portanto, tem-se que a probabilidade de um valor de resíduo padronizado maior que $+2,67$ ser obtido por acaso, sem que exista relação significativamente maior que a média entre os determinantes envolvidos, é menor que $1,0 \%$.

\subsection{Construção do modelo robusto}

Partindo-se das relações significativas entre determinantes identificadas na etapa anterior, é estabelecido o modelo de satisfação de clientes que servirá de base para a construção do modelo robusto.

A construção do modelo robusto segue as seguintes subetapas: 3.5.1 Avaliação dos desvios em relação ao modelo estabelecido; 3.5.2 Ponderação dos respondentes conforme a concordância; 3.5.3 Recontagem ponderada.

Partindo-se dessas novas contagens ponderadas, são identificadas novamente as relações significativas entre determinantes (etapa 3.4) e é construído o diagrama de enlace causal robusto para a satisfação de clientes de serviços.

A avaliação dos desvios em relação ao modelo estabelecido consiste em determinar a concordância de cada um dos questionários (cada um dos respondentes) em relação ao modelo estabelecido (obtido da identificação de relações significativas entre determinantes). Essa concordância é verificada através da soma dos quadrados dos desvios (diferença absoluta entre as respostas fornecidas por um determinado respondente e a resposta esperada, tendo em vista o modelo estabelecido).

Para identificar essa concordância, primeiramente é construída uma matriz correspondente ao modelo estabelecido na qual, para cada relação entre determinantes com resíduos padronizados maiores que +2,67 (critério definido para estabelecer relação estatisticamente significativa), assinala-se o valor de 1 (relação forte existente). Para relações entre determinantes com resíduos padronizados entre $+1,96$ e +2,67 assinala-se o valor 0,5 (dúvida referente à existência de uma relação que possa ser considerada significativa). Para as demais relações que apresentam resíduos padronizados menores que $+1,96$ assinala-se 0 valor 0 (relação significativa inexistente). Dessa forma podem ser testadas não somente as relações estatisticamente significativas, segundo o critério de decisão utilizando a modelagem robusta, mas também relações com valores de desvios próximos ao critério de decisão. No modelo gerado são incorporadas somente as relações significativas fortes, conforme o critério de decisão. É importante destacar que a modelagem robusta também contribui para eliminar relações que não apresentam força significativa suficiente.

O segundo passo consiste em calcular a concordância entre as respostas de cada questionário (onde todas as relações são avaliadas na forma de 0 ou 1) e aquelas previstas na matriz correspondente ao modelo estabelecido. A medida escolhida para avaliar a concordância é proporcional ao inverso do quadrado da soma dos desvios absolutos, conforme apresentado na Equação 2.

$$
C_{i}=\frac{1}{1+\left(\sum A b s_{i}\right)^{2}}
$$

onde:

- $C_{i}$ : índice de concordância de cada questionário, que varia no intervalo $[0,1]$, onde 1 representa concordância plena;

- Abs: diferença absoluta entre as respostas de cada questionário e o que seria esperado considerando o modelo estabelecido.

Uma vez calculados os valores de $C i$, os mesmos são multiplicados por uma constante tal que a soma dos pesos $P i$ resulte igual ao número de respondentes.

$P_{i}=K \times C_{i}$

onde:

- $P_{i}$ é o peso a ser atribuído ao questionário $i$;

- $K=N$ / Somatório dos $P_{i}$;

- $N$ é o tamanho da amostra.

Os valores de $P_{i}$ são utilizados para ponderar cada questionário, de forma que é atribuído um peso maior aos questionários que apresentam maior concordância com o modelo estabelecido. Paralelamente é atribuído um peso menor aos questionários que destoam da maioria. A recontagem ponderada gera uma nova matriz de contagens totais, considerando peso maior para respondentes consistentes e peso menor para aqueles cujas respostas afastam-se das da maioria.

Essas novas contagens ponderadas são utilizadas para identificar as relações significativas entre determinantes, conforme a etapa 3.4 e para, dessa forma, gerar o modelo robusto. 0 procedimento pode ser repetido duas ou três vezes, uma vez que 
a convergência é rápida. A construção do diagrama de causa e efeito é realizada a partir das relações significativas entre as variáveis, indicando o sentido de influência de uma variável sobre outra por meio de uma seta.

\section{Aplicação prática}

Neste trabalho, para a construção do modelo geral de satisfação de clientes de serviços, avaliaram-se 12 segmentos de serviços (restaurante à la carte, teleentrega, escola de idiomas, estação de rádio, salão de beleza, supermercado, serviços médicos, cinema, hotel, serviços odontológicos, farmácia de manipulação e bancos). Esses serviços foram escolhidos para o estudo por contemplarem diferentes categorias de serviços, no que tange a grau tangibilidade e grau de contato e de envolvimento do cliente (LOVELOCK, 1983; SCHMENNER, 1986, 2004; CHASE, 1978, 1981; TINOCO, 2006). As dimensões de grau de tangibilidade, grau de contato com o cliente e de grau de envolvimento do cliente são consideradas entre as mais importantes e mais utilizadas nas tipologias de serviços encontradas na literatura (COOK; GOH; CHUNG, 1999; TINOCO, 2006)

Para cada tipo de serviço utilizou-se um tamanho de amostra de 50 clientes, totalizando 600 respondentes. A pesquisa foi realizada no estado do Rio Grande do Sul e abordou indivíduos com ensino superior concluído e usuários dos serviços avaliados neste estudo. Atendidos esses requisitos, os indivíduos foram selecionados aleatoriamente, de forma a estabelecer uma amostra diversificada no que tange a sexo, idade e classe social. Além disso, antes da aplicação da pesquisa, apresentou-se aos entrevistados uma folha com as definições de cada uma das variáveis envolvidas no estudo, de forma a homogeneizar os conceitos. Esses critérios foram definidos devido à complexidade da terminologia usada na pesquisa e à necessidade de essa população estar familiarizada e consumir os serviços em estudo. A Tabela 1 apresenta as contagens totais utilizadas para a construção do modelo geral de satisfação de clientes de serviços.

Na matriz apresentada na Tabela 1, os valores representam as contagens totais obtidas para a dependência dos determinantes das linhas em relação aos determinantes das colunas.

Antes da aplicação da análise de resíduos padronizados foi realizado o teste de qui-quadrado. Como resultado, foi rejeitada a hipótese nula de independência das variáveis, comprovando a existência de relações entre os determinantes da satisfação.

Para identificar as relações significativas do modelo estabelecido, realizou-se a análise de resíduos padronizados, utilizando a equação 1. Relações significativas foram definidas como aquelas em que a contagem é significativamente maior que a contagem média observada ao longo de todas as células da matriz. As relações significativas com valores de desvio acima de $+2,67$ foram assinaladas com o valor 1,0 na matriz do modelo estabelecido (Tabela 2). Ao mesmo tempo, as relações entre determinantes que apresentaram resíduos padronizados entre $+1,96 \mathrm{e}$ $+2,67$ foram assinaladas com o valor 0,5. Para as demais, que apresentaram resíduos padronizados

Tabela 1. Contagens totais para os serviços pesquisados*.

\begin{tabular}{|c|c|c|c|c|c|c|c|c|c|c|}
\hline \multirow{2}{*}{\multicolumn{2}{|c|}{ Contagens totais }} & \multicolumn{9}{|c|}{ Variáveis independentes } \\
\hline & & $\Xi$ & $\tilde{0}$ & $\sum_{\pi}^{\infty}$ & $气$ & $\frac{\mathscr{T}}{\frac{\pi}{0}}$ & 8 & t & 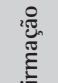 & శ్ల్ల్లి \\
\hline \multirow{9}{*}{$\begin{array}{c}\text { Variáveis } \\
\text { dependentes }\end{array}$} & Imagem & & 080 & 137 & 119 & 403 & 131 & 156 & 212 & 371 \\
\hline & Desejos & 133 & & 318 & 232 & 135 & 088 & 082 & 144 & 193 \\
\hline & Expectativas & 315 & 329 & & 101 & 176 & 151 & 113 & 122 & 172 \\
\hline & Emoções & 059 & 307 & 229 & & 296 & 065 & 093 & 269 & 309 \\
\hline & Qualidade & 135 & 125 & 198 & 184 & & 114 & 234 & 338 & 392 \\
\hline & Preço & 182 & 069 & 094 & 042 & 341 & & 289 & 114 & 215 \\
\hline & Valor & 095 & 137 & 167 & 111 & 372 & 257 & & 263 & 373 \\
\hline & Desconfirmação & 062 & 192 & 282 & 197 & 310 & 072 & 153 & & 441 \\
\hline & Satisfação & 068 & 212 & 227 & 247 & 391 & 118 & 235 & 444 & \\
\hline
\end{tabular}

*Serviços pesquisados: restaurante à la carte, tele-entrega, escola de idiomas, estação de rádio, salão de beleza, supermercado, serviços médicos, cinema, hotel, serviços odontológicos, farmácia de manipulação e bancos. 
Tabela 2. Matriz do modelo estabelecido.

\begin{tabular}{|c|c|c|c|c|c|c|c|c|c|c|}
\hline \multirow{2}{*}{\multicolumn{2}{|c|}{ Modelo estabelecido }} & \multicolumn{9}{|c|}{ Variáveis independentes } \\
\hline & & \multirow[t]{2}{*}{ 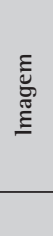 } & \multirow{2}{*}{$\begin{array}{l}\tilde{\mathscr{\Xi}} \\
\tilde{\tilde{\Xi}} \\
0\end{array}$} & \multirow{2}{*}{ 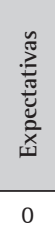 } & \multirow{2}{*}{ 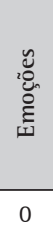 } & \multirow{2}{*}{ 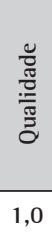 } & \multirow{2}{*}{ 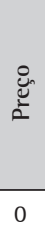 } & \multirow{2}{*}{$\begin{array}{l}\frac{\frac{5}{\pi}}{\pi} \\
0\end{array}$} & \multirow[t]{2}{*}{ 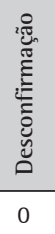 } & \multirow{2}{*}{ 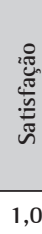 } \\
\hline \multirow{9}{*}{$\begin{array}{c}\text { Variáveis } \\
\text { dependentes }\end{array}$} & Imagem & & & & & & & & & \\
\hline & Desejos & 0 & & 1,0 & 0,5 & 0 & 0 & 0 & 0 & 0 \\
\hline & Expectativas & 1,0 & 1,0 & & 0 & 0 & 0 & 0 & 0 & 0 \\
\hline & Emoções & 0 & 1,0 & 0,5 & & 1,0 & 0 & 0 & 1,0 & 1,0 \\
\hline & Qualidade & 0 & 0 & 0 & 0 & & 0 & 0,5 & 1,0 & 1,0 \\
\hline & Preço & 0 & 0 & 0 & 0 & 1,0 & & 1,0 & 0 & 0 \\
\hline & Valor & 0 & 0 & 0 & 0 & 1,0 & 1,0 & & 1,0 & 1,0 \\
\hline & Desconfirmação & 0 & 0 & 1,0 & 0 & 1,0 & 0 & 0 & & 1,0 \\
\hline & Satisfação & 0 & 0 & 0,5 & 1,0 & 1,0 & 0 & 0,5 & 1,0 & \\
\hline
\end{tabular}

menores que $+1,96$, assinalou-se o valor 0 . Dessa forma obteve-se a matriz do modelo estabelecido, apresentada na Tabela 2 .

Para determinar a concordância dos questionários em relação ao modelo estabelecido, calculou-se a soma dos quadrados dos desvios, a partir da Equação 2. A seguir, os pesos para cada questionário foram calculados a partir da Equação 3. Assim, foi possivel gerar uma nova matriz contendo contagens robustas, onde os questionários de maior concordância em relação ao modelo estabelecido possuem uma maior ponderação em relação aos outros. A Tabela 3 apresenta a matriz de recontagem ponderada utilizada para gerar o modelo robusto para a satisfação de clientes de serviços.

Partindo das contagens ponderadas, as relações significativas entre os determinantes da satisfação de clientes foram identificadas utilizando-se a Equação 1. A Tabela 4 apresenta os valores obtidos de resíduos padronizados para cada relação entre determinantes, a partir das contagens robustas. Utilizou-se como critério de decisão na aceitação de relação significativa entre determinantes valores de desvio acima de $+2,67$. Esse valor está associado a um nível de confiança de 99\%.

Comparando-se a matriz do modelo estabelecido (Tabela 2), representando as relações significativas entre determinantes obtidas da modelagem tradicional (assinaladas com valor de 1,0), com as relações significativas (destacadas em negrito) obtidas da modelagem robusta (Tabela 4), pode-se observar que todas as relações estatisticamente significativas obtidas da modelagem tradicional (desvios acima de $+2,67$ ) foram confirmadas na modelagem robusta. Por outro lado, uma das relações que inicialmente não havia sido detectada como significativa, pois o valor do resíduo padronizado estava no intervalo entre $+1,96$ e $+2,67$, foi confirmada pelo procedimento robusto. A modelagem robusta indicou que os desejos pessoais são afetados pelas emoções vivenciadas, e esta relação foi incorporada ao modelo de satisfação de clientes de serviços.

A Figura 2 apresenta o diagrama causal das relações entre determinantes da satisfação de clientes obtido a partir da modelagem robusta. 0 diagrama construído representa o modelo geral de satisfação clientes para os serviços avaliados.

A influência das respostas afetivas nas atitudes dos clientes é importante principalmente no contexto de serviços devido a sua natureza experimental (CARO; GARCíA, 2007). Em serviços, as emoções associadas com a experiência de consumo desempenham um papel importante na avaliação do cliente em relação à prestação de serviço, na percepção de qualidade e na sua satisfação (OLIVER; RUST; VARKI, 1997; JIANG; WANG, 2006; CARO; GARCíA, 2007). Isso evidencia-se especialmente no contexto de serviços hedônicos, onde a experiência de consumo está determinada por aspectos emotivos e multissensoriais (JIANG; WANG, 2006), como é o caso de alguns serviços avaliados neste trabalho (e.g., cinema, rádio e restaurante à la carte). A relação obtida entre emoções vivenciadas pelos clientes e desejos pessoais através da modelagem robusta confirma a importância dessas respostas emocionais na contextualização da satisfação de clientes de serviços, uma vez que além da influência direta das emoções sobre a satisfação de clientes, as emoções vivenciadas durante a prestação de serviços afetam os desejos pessoais dos clientes. Os desejos pessoais, por sua vez, geram novas expectativas que, 
Tabela 3. Matriz de contagens totais ponderadas (contagens robustas).

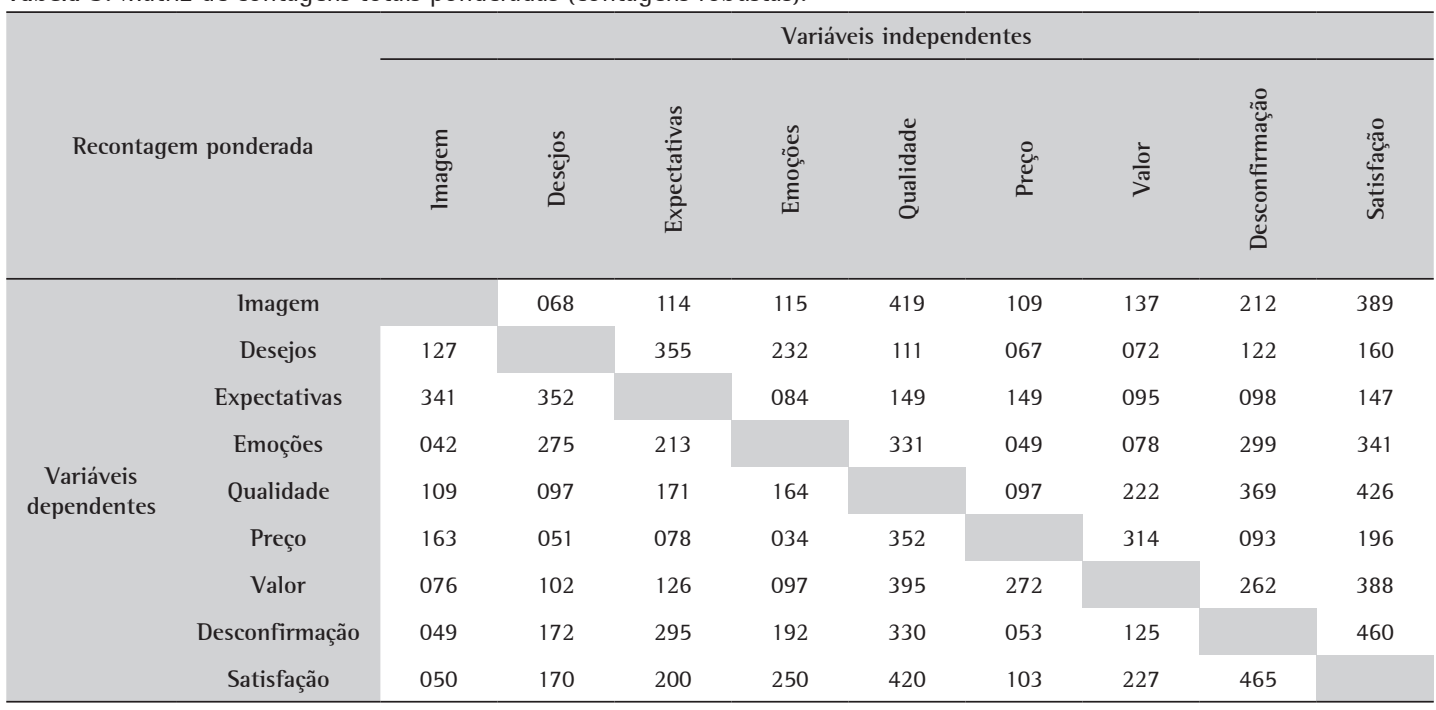

Tabela 4. Análise de resíduos padronizados do modelo robusto.

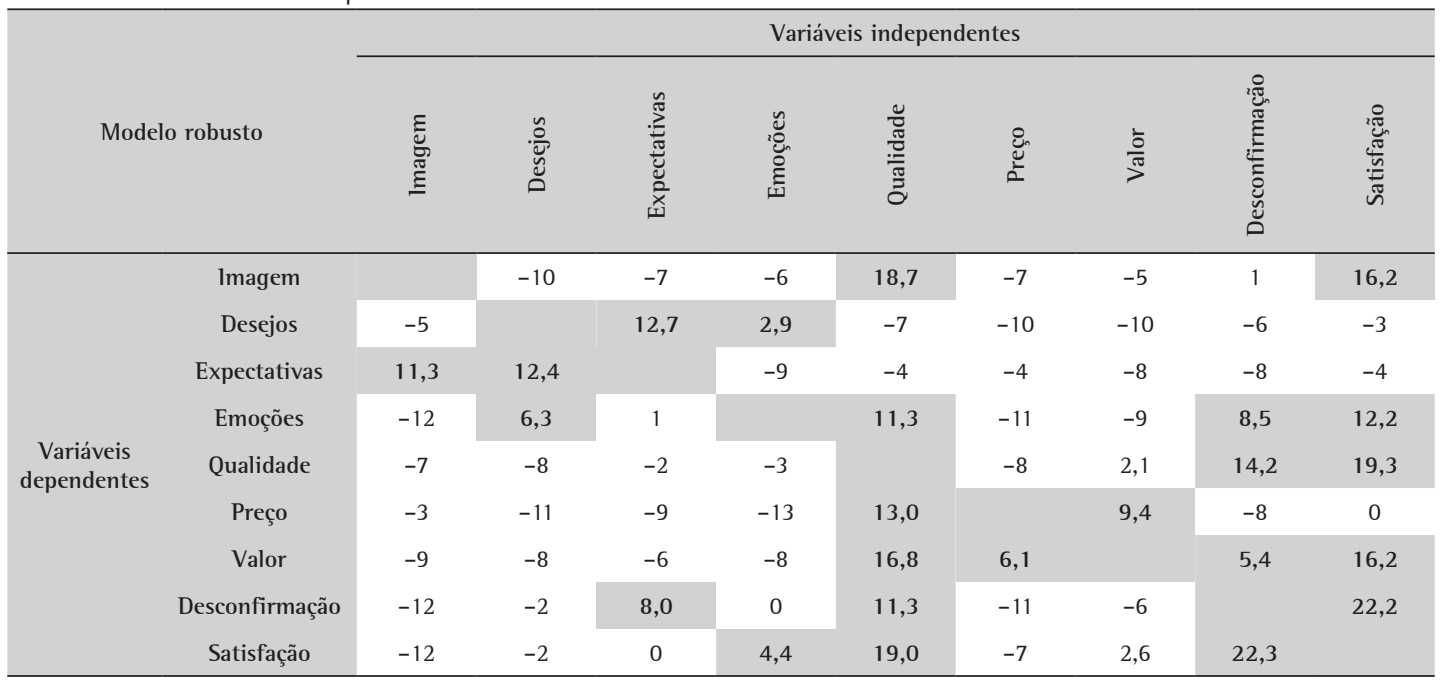

ao serem confirmadas, influenciam a percepção de qualidade e, em consequência, a satisfação dos clientes.

As emoções vivenciadas pelos clientes durante 0 momento da prestação de serviço são influenciadas, por sua vez, pelos desejos dos clientes, pela desconfirmação de expectativas, pela percepção de qualidade em relação ao serviço e pela sua satisfação.

0 modelo robusto para a satisfação de clientes de serviços apresentado na Figura 2 confirma outras relações entre determinantes encontradas na literatura. A desconfirmação de expectativas e a qualidade percebida constituem os principais determinantes da satisfação de clientes (e.g., OLIVER, 1980, 2010; BEl; CHIAO, 2006; BIGNÉ; MATTILA; ANDREU, 2008; LIANG; ZHANG, 2009). Do ponto de vista dos clientes, esses três determinantes estão inter-relacionados e são experimentados simultaneamente. Outros autores também confirmam a existência de ambiguidade ao definir a relação causal entre satisfação de clientes e qualidade percebida (BRADY; ROBERTSON, 2001; NGUYEN; LEBLANC, 1998).

A imagem corporativa das empresas prestadoras de serviços é influenciada principalmente pela percepção da qualidade do serviço e pela satisfação dos clientes, confirmando pesquisas de Nguyen e LeBlanc (1998), Lai, GRIFFIN e BABIN (2009) e Hu, Kandampully e Juwaheer (2009). A imagem corporativa cria novas expectativas nos clientes, validando os achados de Yoon, Guffey e Kijewski (1993), as quais são confirmadas, afetando a percepção de qualidade e satisfação desses clientes. 


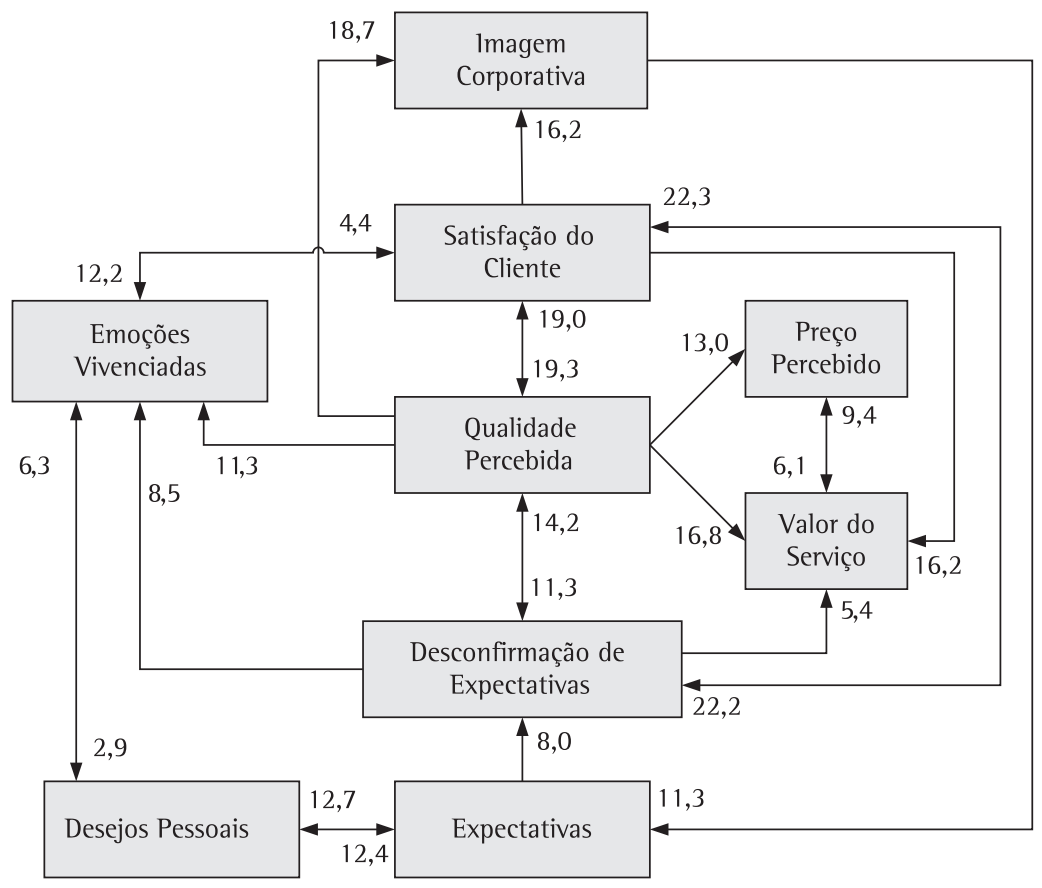

Figura 2. Modelo robusto para a satisfação de clientes de serviços.

0 valor do serviço é visto, do ponto de vista dos clientes de serviços, como uma consequência da desconfirmação de suas expectativas, de sua satisfação e da relação entre os benefícios do serviço (qualidade percebida) e os custos envolvidos (preço percebido). lsso pode ser justificado pela maior dificuldade de os clientes avaliarem o valor dos serviços em relação à avaliação de valor dos produtos (bens tangíveis) (DAY, 2002).

Finalmente, a percepção de preço dos clientes de serviços está influenciada, principalmente, pela qualidade percebida e pelo valor do serviço. Quanto maior o benefício percebido pelo cliente em relação a um serviço, menor a percepção do preço pago. Esse resultado é verificado nas pesquisas de Chen, Gupta e Rom (1994).

\section{Conclusões}

0 presente artigo apresenta a proposição de uma abordagem robusta para a modelagem da satisfação de clientes, a partir da proposta de Tinoco e Ribeiro (2007). A abordagem robusta representa uma extensão da sistemática proposta pelos autores para identificar relações significativas entre determinantes da satisfação de clientes. Os procedimentos robustos utilizados na abordagem proposta neste artigo permitem identificar dados atípicos (respondentes inconsistentes), através da avaliação de um índice de concordância de cada questionário em relação à média das observações, e atribuir uma ponderação menor para esses respondentes considerados discrepantes em relação à maioria dos dados. Dessa forma, os respondentes discrepantes possuem um peso menor na contagem total dos dados e, em consequência, um efeito menor na análise dos resultados gerados.

A abordagem robusta foi testada através da construção de um modelo geral para a satisfação de clientes de serviços. Para isso, foram avaliados 12 segmentos de serviços escolhidos para o estudo por contemplarem diferentes categorias de serviços, no que tange a grau de tangibilidade e grau de contato e de envolvimento do cliente. A abordagem robusta mostrou-se eficiente para a construção desse modelo de satisfação de clientes, uma vez que permitiu confirmar a maioria das relações significativas entre determinantes da satisfação obtidos a partir da abordagem tradicional proposta por Tinoco e Ribeiro (2007) e, além disso, incorporar no modelo outra relação significativa que não seria considerada a partir da técnica tradicional.

Como principais implicações teóricas e práticas podem ser mencionadas a contribuição dos resultados obtidos com as pesquisas desenvolvidas para o setor de serviços, especificamente no campo da satisfação de clientes. Além disso, a abordagem robusta constitui um método para identificar relações significativas entre múltiplos determinantes, a partir da aplicação 
de procedimentos simples, sem requerer tamanhos de amostras excessivos e lidando adequadamente com respondentes atípicos, usualmente associados a falta de interesse ou falta de compreensão do questionário.

Entre as principais limitações do artigo podem ser destacadas (i) a avaliação de alguns segmentos do setor de serviços e (ii) a delimitação geográfica, uma vez que as pesquisas foram conduzidas na cidade de Porto Alegre, com usuários dos diversos segmentos de serviços avaliados. Apesar de os serviços avaliados contemplarem diferentes tipologias (referentes a grau de tangibilidade e grau de contato e de envolvimento do cliente), a generalização para outros tipos de serviços talvez necessite de adaptações. Sugere-se para trabalhos futuros a aplicação da modelagem robusta para outros tipos de serviços.

\section{Referências}

ABRAMOVITZ, L.; MENDES, B. V. M. Sobre a não-existência dos estimadores de máxima verossimilhança: uma aplicação na estimação do risco de crédito. Revista Brasileira de Estatística, v. 61, n. 216, p. 07-30, 2000.

AGRESTl, A.; FINLAY, B. Statistical Methods for the Social Sciences. 4th ed. New Jersy: Pearson Prentice Hall, 2009. 609 p.

ALMA, Ö. G. Comparison of Robust Regression Methods in Linear Regression. International Journal of Contemporary Mathematical Sciences, v. 6, n. 9, p. 409-421, 2011.

ANDERSON, E. W.; FORNELL, C. Foundations of the American Customer Satisfaction Index. Total Quality Management, v. 11, n. 7, p. 869-882, 2000. http:// dx.doi.org/10.1080/09544120050135425

ANDERSON, E. W. et al. Customer Satisfaction, Market Share, and Profitability: Findings from Sweden. Journal of Marketing, v. 58, n. 3, p. 53-66, 1994. http://dx.doi. $\operatorname{org} / 10.2307 / 1252310$

ANDREASSEN, T. W.; LINDESTAD, B. Customer loyalty and complex services - The impact of corporate image on quality, customer satisfaction and loyalty for customers with varying degrees of service expertise. International Journal of Service Industry Management, v. 9. n. 1, p. 7-23, 1998. http://dx.doi. org/10.1108/09564239810199923

ANALYTICAL METHODS COMMITTEE - AMC. Robust Statistics - How Not to Reject Outliers: Part 1. Basic Concepts, Analyst. AMC, 1989. v. 114.

APPLETON-KNAPP, S. L.; KRENTLER, K. A. Measuring Student Expectations and Their Effects on Satisfaction: The Importance of Managing Student Expectations. Journal of Marketing Education, v. 28, p. 254-264, 2006. http://dx.doi.org/10.1177/0273475306293359

BEl, L-T.; CHIAO, Y-C. An Integrated Model for the Effects of Perceived Product, Perceived Service Quality, and Perceived Price Fairness on Consumer Satisfaction and Loyalty. Journal of Consumer Satisfaction, Dissatisfaction and Complaining Behavior, v. 14, 2001.

BEl, L-T.; CHIAO, Y-C. The determinants of customer loyalty: an analysis of intangible factors in three service industries. International Journal of Commerce and
Management, v. 16, n. 3-4, p. 162, 2006. http://dx.doi. org/10.1108/10569210680000215

BIGNÉ, J. E.; MATTILA, A. S.; ANDREU, L. The impact of experiential consumption cognitions and emotions on behavioral intentions. Journal of Services Marketing, v. 22, n. 4, p. 303-315, 2008. http://dx.doi. org/10.1108/08876040810881704

BRADY, M. K.; ROBERTSON, C. J. Searching for a consensus on the antecedent role of service quality and satisfaction: an exploratory cross-national study. Journal of Business Research, v. 51, p. 53-60, 2001. http://dx.doi. org/10.1016/S0148-2963(99)00041-7

BRUHN, M.; GRUND, M. A. Theory, development and implementation of national customer satisfaction indices: the Swiss Index of Customer Satisfaction (SWICS). Total Quality Management, v. 11, n. 7, p. 1017-1028, 2000. http://dx.doi.org/10.1080/09544120050135542

BUSTOS, 0. H. Outliers e Robustez. Revista Brasileira de Estatística, v. 49, n. 192, p. 7-30, 1988.

CARO, L. M.; GARCÍA, J. A. M. Cognitive-affective model of consumer satisfaction. An exploratory study within the framework of a sporting event. Journal of Business Research, v. 60, p. 108-114, 2007. http://dx.doi. org/10.1016/j.jbusres.2006.10.008

CHASE, R. B. Where does the customer fit in a service operation? Harvard Business Review, v. 56, n. 4, p. 13742, 1978. PMid:10239167.

CHASE, R. B. The Customer Contac Approach to Services: Theoretical Bases and Practical Extensions. Operations Research, v. 24, n. 4, p. 698-706, 1981. http://dx.doi. org/10.1287/opre.29.4.698

CHEN, 1. J.; GUPTA, A.; ROM, W. A study of price and quality in service operations. International Journal of Service Industry Management, v. 5, n. 2, p. 23-33, 1994. http:// dx.doi.org/10.1108/09564239410057663

$\mathrm{CHOl}, \mathrm{K} .-\mathrm{S}$. et al. The relationships among quality, value, satisfaction and behavioral intention in health care provider choice: A South Korean study. Journal of Business Research, v. 57, p. 913-921, 2004. http://dx.doi. org/10.1016/S0148-2963(02)00293-X

CHURCHILL JUNIOR, G. A.; SURPRENANT, C. An Investigation into the Determinants of Customer Satisfaction. Journal of Marketing Research, v. 19, p. 491-504, 1982. http:// dx.doi.org/10.2307/3151722

COLLIER, J. E.; BIENSTOCK, C. C. Measuring Service Quality in E-Retailing. Journal of Service Research, v. 8, p. 260-275, 2006. http://dx.doi. org/10.1177/1094670505278867

COOK, D. P.; GOH, C-H.; CHUNG, C. H. Service Typologies: A State of the Art Survey. Production and Operations Management, v. 8, n. 3, 1999

CRONIN JUNIOR, J. J.; BRADY, M. K.; HULT, T. G. Assessing the effects of quality, value and customer satisfaction on consumer behavioral intentions in service environments. Journal of Retailing, v. 76, n. 2, p. 193-218, 2000. http:// dx.doi.org/10.1016/S0022-4359(00)00028-2

DAY, E. The Role of Value in Consumer Satisfaction. Journal of Consumer Satisfaction, Dissatisfaction and Complaining Behavior, v. 15, p. 22-32, 2002

FORNELL, C.; JOHNSON, M. D.; ANDERSON, E. W. The American Customer Satisfaction Index: Nature, purpose, and findings. Journal of Marketing, v. 60, n. 4, p. 7-12, 1996. http://dx.doi.org/10.2307/1251898 
GOSLING, M.; GONÇALVES, C. A. Modelagem por Equações Estruturais: conceitos e aplicações. Revista FACES - Revista de Administração, v. 2, n. 2, p. 8395, 2003.

GRÖNROOS, C. Service Management and Marketing: customer management in service competition. 3rd ed. Chichester: John Wiley \& Sons Ltd, 2007. 483 p.

HERRMANN, A. et al. The influence of price fairness on customer satisfaction: an empirical test in the context of automobile purchases. Journal of Product \& Brand Management, v. 16, n. 1, p. 49-58, 2007. http://dx.doi. org/10.1108/10610420710731151

HOMBURG, C.; HOYER, W.; KOSCHATE, N. Customers' Reactions to Price Increases: do customer satisfaction and perceived motive fairness matter? Journal of the Academy of Marketing Science, v. 33, n. 1, p. 3649, 2005. http://dx.doi.org/10.1177/0092070304269953

HU, H.-H.; KANDAMPULLY, J.; JUWAHEER, T. D. Relationships and impacts of service quality, perceived value, customer satisfaction, and image: an empirical study. The Service Industries Journal, v. 29, n. 2, p. 111-125, 2009. http:// dx.doi.org/10.1080/02642060802292932

HUBER, P. J.; RONCHETTl, E. M. Robust Statistics. 2nd ed. New Jersey: John Wiley \& Sons, 2009. 354 p. http:// dx.doi.org/10.1002/9780470434697

JIANG, Y.; WANG, C. L. The impact of affect on service quality and satisfaction: the moderation of service contexts. Journal of Services Marketing,v. 20, n. 4, p. 211-218, 2006. http://dx.doi.org/10.1108/08876040610674562

JOHNSON, M. D.; GUSTAFSSON, A. Improving Customer Satisfaction, Loyalty, and Profit: an integrated measurement and management system. San Francisco: Jossey-Bass, 2000. $214 \mathrm{p}$.

JOHNSON, M. D.; NADER, G.; FORNELL, C. Expectations, perceived performance, and customer satisfaction for a complex service: The case of bank loans. Journal of Economic Psychology, v. 17, p. 163-182, 1996. http:// dx.doi.org/10.1016/0167-4870(96)00002-5

JUN, S.; HYUN, Y. J.; GENTRY, J. W. The Relative Influence of Affective Experience on Consumer Satisfaction under Positive versus Negative Discrepancies. Journal of Consumer Satisfaction, Dissatisfaction and Complaining Behavior, v. 14, p. 141-153, 2001.

KLEINBAUM, D. G. et al. Applied Regression Analysis and Other Multivariable Methods. 4th ed. Thomson Learning, 2008. $906 \mathrm{p}$.

KLEINBAUM, D.; KUPPER, L. Applied regression analysis and other multivariable methods. Wadsworth Publishing Company, 1978. 556 p.

LARÁN, J. A.; ALMEIDA, S. 0.; HOFFMANN, J. A. Desejos dos consumidores: um olhar sobre os determinantes da satisfação. Revista de Administração, v. 39, n. 1, p. 4252, 2004.

LAI, F.; GRIFFIN, M.; BABIN, B. J. How quality, value, image, and satisfaction create loyalty at a Chinese telecom. Journal of Business Research, v. 62, p. 980-986, 2009. http://dx.doi.org/10.1016/j.jbusres.2008.10.015

LIANG, X.; ZHANG, S. Investigation of customer satisfaction in student food service. International Journal of Quality and Service Sciences, v. 1, n. 1, p. 113-124, 2009. http:// dx.doi.org/10.1108/17566690910945903
LOVELOCK, C. H. Classifying Services to Gain Strategic Marketing Insights. Journal of Marketing, v. 47, p. 9-20, 1983. http://dx.doi.org/10.2307/1251193

MARTIN, D. et al. The role of emotion in explaining consumer satisfaction and future behavioural intention. Journal of Services Marketing, v. 22, n. 3, p. 224-236, 2008. http:// dx.doi.org/10.1108/08876040810871183

MEYERS, L. S.; GAMST, G.; GUARINO, A. J. Applied Multivariate Research: Design and Interpretation. Sage Publications, 2006. 722 p.

MONTGOMERY, D. C.; PECK, E. A.; VINING, G. Introduction to Linear Regression Analysis. 4th ed. Wiley, 2006. 640 p.

MORGAN, N. A.; REGO, L. L. The Value of different customer satisfaction and loyalty metrics in predicting business performance. Marketing Science, v. 25, n. 5, p. 426439, 2006. http://dx.doi.org/10.1287/mksc. 1050.0180

NGUYEN, N.; LEBLANC, G. The mediating role of corporate image on customers'retention decisions: an investigation in financial services. International Journal of Bank Marketing, v. 16, n. 2, p. 52-65, 1998. http://dx.doi. org/10.1108/02652329810206707

OLIVER, R. L. A Cognitive Model of the Antecedents and Consequences of Satisfaction Decisions. Journal of Marketing Research, v. 17, p. 460-469, 1980. http:// dx.doi.org/10.2307/3150499

OLIVER, R. L. Cognitive, Affective, and Attribute Bases of the Satisfaction Response. Journal of Consumer Research, v. 20, p. 418-430, 1993. http://dx.doi. org/10.1086/209358

OLIVER, R. L.; RUST, R.; VARKI, S. Customer Delight: foundations, findings, and managerial insights. Journal of Retailing, v. 73, n. 3, p. 311-336, 1997. http://dx.doi. org/10.1016/S0022-4359(97)90021-X

OLIVER, R. L. Satisfaction: a behavioral perspective on the consumer. 2nd ed. New York: M.E. Sharpe, 2010. 519 p.

OLSHAVSKY, R. W.; KUMAR, A. Revealing the Actual Roles of Expectations in Consumer Satisfaction with Experience and Credence Goods. Journal of Consumer Satisfaction, Dissatisfaction and Complaining Behavior, v. 14, p. 6073, 2001.

PATTERSON, P. G.; JOHNSON, L. W.; SPRENG, R. A. Modeling the Determinants of Customer Satisfaction for Business to Business Professional Services. Journal of the Academy of Marketing Science, v. 25, n. 1, p. 4-17, 1997. http:// dx.doi.org/10.1007/BF02894505

SCHMENNER, R. W. How can service businesses survive and prosper? Sloan Management Review, v. 28, n. 3, p. 2132, 1986

SCHMENNER, R. W. Service Businesses and Productivity. Decision Sciences, v. 35, n. 3, p. 333-347, 2004. http:// dx.doi.org/10.1111/j.0011-7315.2004.02558.x

SPRENG, R. A.; MACKENZIE, S. B.; OLSHAVSKY, R. W. A reexamination of the determinants of consumer satisfaction. Journal of Marketing, v. 60, n. 3, p. 1532, 1996. http://dx.doi.org/10.2307/1251839

SPRENG, R. A.; MACKOY, R. D. An Empirical Examination of a Model of Perceived Service Quality and Satisfaction. Journal of Retailing, v. 72, n. 2, p. 201-214, 1996. http://dx.doi.org/10.1016/S0022-4359(96)90014-7

SZYMANSKI, D. M.; HENARD, D. H. Customer Satisfaction: A Meta-Analysis of the Empirical Evidence. Journal of 
the Academy of Marketing Science, v. 29, n. 16, p. 1635, 2001.

TINOCO, M. A. C. Proposta de modelos de satisfação dos consumidores de serviços. 2006. 119 f. Dissertação (Mestrado em Engenharia de Produção)-Universidade Federal do Rio Grande do Sul, Porto Alegre, 2006.

TINOCO, M. A. C.; RIBEIRO, J. L. D. Uma nova abordagem para a modelagem das relações entre os determinantes da satisfação dos clientes de serviços. Produção, v. 17, n. 3, p. 454-470, 2007. http://dx.doi.org/10.1590/ S0103-65132007000300005

TSE, D. K.; WILTON, P. C. Models of Consumer Satisfaction Formation: An Extension. Journal of Marketing Research, v. 25, p. 204-212, 1988. http://dx.doi. $\operatorname{org} / 10.2307 / 3172652$

TSIROS, M.; MITTAL, V.; ROSS JUNIOR, W. The Role of Attributions in Customer Satisfaction: A Reexamination. Journal of Consumer Researchn, v. 31, n. 2, p. 476483, 2004. http://dx.doi.org/10.1086/422124

VAN RYZIN, G. G. Testing the Expectancy Disconfirmation Model of Citizen Satisfaction with Local Government. Journal of Public Administration Research and Theory, v. 15, 2005.
VLACHOS, P. A.; VRECHOPOULOS, A. P. Determinants of behavioral intentions in the mobile internet services market. Journal of Services Marketing, v. $22, \quad$ n. 4 , p. 280-291, 2008. http://dx.doi. org/10.1108/08876040810881687

WANG, Y.; LO, H. Service quality, customer satisfaction and behavior intentions: evidence from China's telecommunication industry. Info - The journal of policy, regulation and strategy for telecommunications, v. 4, n. 6, p. 50-60, 2002.

WHITTAKER, G.; LEDDEN, L.; KALAFATIS, S. P. A reexamination of the relationship between value, satisfaction and intention in business services. Journal of Services Marketing, v. 21, n. 5, p. 345-357, 2007. http:// dx.doi.org/10.1108/08876040710773651

$\mathrm{Yl}$, Y. The determinants of consumer satisfaction: the moderating role of ambiguity. Advances in Consumer Research, v. 20, p. 502-506, 1993.

YOON, E.; GUFFEY, H. J.; KIJEWSKl, V. The Effects of Information and Company Reputation on Intentions to Buy a Business Service. Journal of Business Research, v. 27, p. 215-228, 1993.

\title{
Modeling customer satisfaction determinants in services: a robust approach
}

\begin{abstract}
This paper presents a robust approach for modeling customer satisfaction in services. This approach represents an extension of the method proposed by Tinoco and Ribeiro (2007), through the inclusion of robust statistics that can adequately represent the causal relations involving customer satisfaction in a service environment, even in the presence of outliers (inconsistent respondents). These robust procedures allow one to identify inconsistent respondents and to assign a weight according to the agreement of each questionnaire based on the average of the observations. The approach proposed in this work is demonstrated by building a general model of customer satisfaction in services. For this purpose, twelve segments of services, chosen to represent different service types with respect to tangibility, customer contact and customer involvement degree, were assessed. The robust approach confirmed the most significant relationships between determinants of satisfaction obtained from the traditional approach proposed by Tinoco and Ribeiro (2007) and also incorporated a further important relationship that would not be considered from the traditional technique.
\end{abstract}

\section{Keywords}

Robust modeling. Customer satisfaction. Services. 